\title{
Brain Metastases and Breast Cancer Subtypes
}

\author{
Ana M. Gonzalez-Angulo ${ }^{\mathrm{a}, \mathrm{b}}$ Gabriel N. Hortobagyi ${ }^{\mathrm{a}}$ \\ ${ }^{a}$ Department of Breast Medical Oncology, \\ ${ }^{\mathrm{b}}$ Department of Systems Biology, The University of Texas M. D. Anderson Cancer Center, Houston, TX, USA
}

Breast cancer is a heterogeneous disease characterized by a wide spectrum of clinical, pathological and molecular characteristics that composed a number of recognized biological subtypes which have differing prognostic profiles [1-4]. Although at least 5 molecular subtypes of breast cancer have been identified by gene expression profiles, using clinical and basic histologic assays, and for therapy selection, breast cancer is classified into hormone receptor (HR)-positive/HER2negative, HER2-positive and triple receptor negative (TNBC) disease. It is also recognized that the TNBC and HER2-positive subtypes are associated with particularly poor outcomes compared to the HR-positive subtype [1-4]. The incidence of brain metastases in patients with metastatic breast cancer is reported to range from 10 to $16 \%$, approaching $30 \%$ when autopsy diagnosis of brain metastases is included [5-7]. Brain metastases generally tend to occur late in the course of metastatic breast cancer and are typically associated with 1 - and 2 -year survival rates of only $20 \%$ and $<2 \%$, respectively $[8,9]$. Factors that have been reported to be associated with a higher risk of developing brain metastases include young age, four or more positive lymph nodes, high tumor grade and HER-2 overexpression [5, 10-13]. In addition, several studies have reported a greater propensity of estrogen receptor-negative tumors metastasizing to the brain compared to estrogen receptor-positive tumors $[10,12,14]$.

In this issue of the journal, Kwon et al. [15], retrospectively reviewed 66 patients with breast cancer and brain metastasis and found that the largest proportion of their series (39.4\%) had TNBC. As expected, patients with TNBC and HER2positive disease had shorter disease-free intervals, and the median time intervals from distant metastasis to development of brain metastasis were 20.6, 19.5, and 9.0 months in patients with HR-positive, HER2-positive and TNBC, respectively $(\mathrm{p}=0.012)$. Further, the times from initial breast cancer diagnosis to brain metastasis were 52.9, 33.6, and 25.5 months for patients with HR-positive, HER2-positive and TNBC, respectively $(\mathrm{p}=0.026)$. However, overall survival rates did not dif- fer significantly. Although this might be a good opportunity to review the association of TNBC and HER-positive tumors with brain metastases, it is difficult to interpret the data without having the denominators for each breast cancer subtype and see the true incidence of brain metastases in each group.

With the known overall poor prognostic outcome associated with TNBC, and the inference from retrospective studies that this subtype may have an increased propensity to develop brain metastases, which in self is associated with poor prognosis across all types of breast tumors, our group studied the incidence of brain metastases in a cohort of women with early stage TNBC and evaluated their survival outcomes [16]. We found that among 679 patients with TNBC, 42 (6.2\%) patients developed brain metastases of whom $24(3.5 \%)$ had brain metastases as the first metastatic site. Median survival for all patients who developed brain metastases was 2.9 months. Several studies have reported an associated higher incidence of brain metastases in women with HR-negative breast cancer compared to those with HR-positive disease. In one of the earlier studies to look at the issue, Samaan et al. [14] reported on a cohort of 217 women with primary breast cancer and found that women whose tumors were estrogen receptor (ER)-negative were more likely to metastasize to the brain compared to women whose tumors were ER-positive (10\% vs. $4 \%$ ). Using a cohort of over 10,000 women Tham et al. [12] noted similar results with women whose tumors were ERnegative having higher odds of developing brain metastases (OR $=2.8,95 \%$ CI 2.1-3.7, $\mathrm{p}<0.001)$.

With the introduction of more efficacious chemotherapeutic and targeted agents for metastatic breast cancer, control of systemic disease has improved leading to concerns of increase in the development of brain metastases. This may be particularly true for patients with HER2-positive metastatic breast cancer. With the introduction of trastuzumab, the natural history of HER2-positive metastatic breast cancer changed and several studies evaluating patients with metastatic HER2positive breast cancer receiving front-line trastuzumab-based

\section{KARGER}

Fax +497614520714

Information@Karger.de

www.karger.com (c) 2010 S. Karger GmbH, Freiburg

Accessible online at:

www.karger.com/onk
Ana M. Gonzalez-Angulo M.D, M.Sc.

Department of Breast Medical Oncology, Unit 1354

The University of Texas M. D. Anderson Cancer Center

1515 Holcombe Boulevard, Houston, TX 77030, USA

Tel. +1 713 792-2817, Fax -4385

agonzalez@mdanderson.org 
therapeutic regimens reported high incidences of CNS metastases, higher than those reported historically. Interestingly, Dawood et al. [17] reported that patients with HER2positive disease treated with trastuzumab had longer times to development of and better survival from CNS metastases compared to patients with HER2-positive disease who never received trastuzumab and patients with HER2-negative breast cancer.

The work done in identifying risk factors associated with the development of brain metastases has been mainly undertaken in an effort to identify patients at high risk of developing brain metastases and who would benefit from early detec- tion and/or preventive measures such as prophylactic cranial radiation therapy or the use of agents that specifically cross the blood-brain barrier in the adjuvant setting. This study adds to other published data and provides provocative evidence and strong motivation to study these populations in clinical trials and assess the value of preventive measures against the development of brain metastases.

\section{Conflict of Interest}

Both authors declare that they have no conflicts of interests.

\section{References}

1 Perou CM, Sorlie T, Eisen MB, et al.: Molecular portraits of human breast tumors. Nature 2000:406: $747-752$.

2 Sorlie T: Molecular portraits of breast cancer: Tumour subtypes as distinct disease entities. Eur J Cancer 2004;40:2667-2675.

3 Sorlie T, Perou CM, Tibshirani R, et al.: Gene expression patterns of breast carcinomas distinguish tumor subclasses with clinical implications. Proc Natl Acad Sci U S A 2001;98:10869-10874.

4 Brenton JD, Carey LA, Ahmed AA, et al.: Molecular classification and molecular forecasting of breast cancer: Ready for clinical application? J Clin Oncol 2005;23:7350-7360.

5 Lin NU, Bellon JR, Winer EP: CNS metastases in breast cancer. J Clin Oncol 2004;1;22:3608-3617.

6 Tsukada Y, Fouad A, Pickren JW, Lane WW: Central nervous system metastasis from breast carcinoma. Autopsy study. Cancer 1983;52:2349-2354.

7 Patanaphan V, Salazar OM, Risco R: Breast cancer: metastatic patterns and their prognosis. Southern Medical Journal 1988:81:1109-1112.
8 DiStefano A, Yap HY, Hortobagyi GN, et al.: The natural history of breast cancer patients with brain metastases. Cancer 1979;44:1913-1918.

$\checkmark 9$ Engel J, EcKel R, Aydemir U, et al.: Determinants and prognoses of locoregional and distant progression in breast cancer. Int J Radiat Oncol Biol Phys 2003;55:1186-1195.

10 Pestalozzi BC, Zahrieh D, Price KN, et al.: Identifying breast cancer patients at risk for central nervous system (CNS) metastases in trials of the International Breast Cancer Study Group (IBCSG). Ann Oncol 2006;17:935-944.

11 Hicks DG, Short SM, Prescott NL, Tarr SM, Coleman KA, Yoder BJ, et al.: Breast cancers with brain metastases are more likely to be estrogen receptor negative, express the basal cytokeratin CK 5/6, and overexpress HER2 or EGFR. Am J Surg Pathol 2006;30:1097-1104.

12 Tham YL, Sexton K, Kramer R, Hilsenbeck S, Elledge R: Primary breast cancer phenotypes associated with propensity for central nervous system metastases. Cancer 2006;107:696-704.
13 Stemmler HJ, Kahlert S, Siekiera W, Untch M, Heinrich B, Heinemann V: Characteristics of patients with brain metastases receiving trastuzumab for HER2 overexpressing metastatic breast cancer. Breast 2006;15,219-225.

14 Samaan NA, Buzdar AU, Aldinger KA et al.: Estrogen receptor: A prognostic factor in breast cancer. Cancer 1981;47:554-560.

15 Kown HC, Oh SY, Kim SH, et al.: Clinical outcomes and breast cancer subtypes in patients with brain metastases. Onkologie 2010;33: DOI: 10.1159/ 000286281

$16 \overline{\text { Dawood S }}$, Broglio K, Esteva FJ, et al.: Survival among women with triple receptor-negative breast cancer and brain metastases. Ann Oncol 2009;20: 621-627.

17 Dawood S, Broglio K, Esteva FJ, et al.: Defining prognosis for women with breast cancer and CNS metastases by HER2 status. Ann Oncol 2008;19: $1242-1248$ 